Check for updates

Cite this: RSC Adv., 2019, 9, 17440

\title{
SiBaoChongCao exhibited anti-fatigue activities and ameliorated cancer cachexia in mice
}

\author{
Qiang Shen, ${ }^{a}$ Chun-Xiao Miao, ${ }^{a}$ Wan-Li Zhang, ${ }^{a}$ Yi-Wei Li, ${ }^{a}$ Qiao-Qiao Chen, ${ }^{b}$ \\ Xiao-Xiang Li, ${ }^{\mathrm{C}}$ Xuan Liu*b and Xiong-Wen Zhang (iD *a
}

SiBaoChongCao (SBCC) is a functional food product containing fermentation of Acremonium terricola belonging to the Cordyceps genus. SBCC at 1 and $2 \mathrm{~g} \mathrm{~kg}^{-1}$ for 20 days exhibited anti-fatigue activities such as increasing exhaustive swimming and running time of mice and increasing the strength of muscle. The increased muscle endurance in SBCC-treated mice might be related to enhancement of muscle cell growth and differentiation and improvement of muscle response to exercise training, as shown by an increase in muscle cross-sectional area and elevation of MHC, MyoD, MyoG and PGC-1 $\alpha$ levels. And, SBCC at $1.5 \mathrm{~g} \mathrm{~kg}^{-1}$ could ameliorate cancer-related cachexia such as ameliorating decrease in body temperature and inhibiting fat tissue atrophy. The anti-cachexia effects of SBCC might be related to inhibition of inflammatory cytokine IL- 6 secretion and suppression of over-lipolysis and lipid overutilization through inhibiting the activation of AMPK and p38 MAPK and down-regulating the level of UCP1.

Received 27th January 2019

Accepted 21st May 2019

DOI: 10.1039/c9ra00718k

rsc.li/rsc-advances

amino acids were measured as 84.50, 44.60, 0.432, 0.5965 and $218.1 \mathrm{~g} \mathrm{~kg}^{-1}$, respectively. ${ }^{6} \mathrm{SBCC}$ is now popularly used in China as a functional food for people with poor health and is also taken by patients with consumptive diseases such as cancer. To further check and understand the activities of SBCC, antifatigue and anti-cachexia activities of SBCC and the possible mechanisms were investigated in the present study.

Firstly, the anti-fatigue activities of SBCC were checked. Fatigue, defined as a situation in which the capacity for work is diminished and efficiency of accomplishment reduced, includes both mental and physical fatigue. ${ }^{7,8}$ Approaches such as regular exercise, a balanced diet, and complementary and alternative medicines (functional foods) have been shown to be helpful for improving fatigue. In the present study, two classic fatigue animal models, exhaustive swimming test and exhaustive running test, were used to evaluate the anti-fatigue capacity of SBCC. And, the influence of SBCC on muscle strength was also checked.

Secondly, we tried to check the potency of SBCC in ameliorating cancer cachexia. Cachexia is a serious consumptive syndrome that occurs during many chronic diseases, including cancer. Cancer cachexia is mainly characterized by fatigue, loss of muscle and fat mass, excessive consumption of energy and systemic inflammation. According to the statistics, more than $50 \%$ of advanced cancer patients were affected by cachexia and cachexia accounted for nearly $30 \%$ of cancer-related deaths. ${ }^{\mathbf{9}, 10}$ However, because of the complex and unclear pathogenesis of cancer cachexia, there is no FDA-approved drug for treatment of cancer cachexia up to now. Therefore, methods such as nutrition supplementation, anti-inflammation, and appetite stimulation are currently used to alleviate cancer cachexia. Notably,

${ }^{a}$ Shanghai Engineering Research Center of Molecular Therapeutics and New Drug Development, College of Chemistry and Molecular Engineering, East China Normal University, Shanghai 200062, China. E-mail: xwzhang@sat.ecnu.edu.cn; Tel: +86 21 52127904

${ }^{b}$ Institute of Interdisciplinary Integrative Biomedical Research, Shanghai University of Traditional Chinese Medicine, Shanghai 201203, China. E-mail: 13764960370@163. com; Tel: +862151323192

${ }^{c}$ Hefei SiBaoChongCao Technology Co. Ltd, Hefei 230088, China 
complementary and alternative medicines including traditional Chinese medicines and natural herbal medicines have been used in the treatment of cancer cachexia and exhibited promising results. ${ }^{11,12}$ However, as far as we know, no study checking the effects of Cordyceps fungi, either natural or fermented, has been reported. Thus, in the present study, we systematically observed effects of SBCC on cancer cachexia in C26 tumorbearing mice in vivo. The food intake, body weight, body temperature, tumor growth, fat mass and muscle mass of the C26 tumor-bearing mice with or without SBCC treatment were observed. And, the histological characteristics of tissues and expression of signal proteins in tissues were also observed.

Taken together, the global aim of this study is to assess the efficacy of SBCC, a functional food containing fermented Cordyceps militaris, as a complementary treatment of fatigue and cancer cachexia.

\section{Material and methods}

\section{Materials}

SBCC was provided by Hefei SiBaoChongCao Technology Co. Ltd (Hefei, PR China). RPMI-1640, penicillin/streptomycin and trypsin/EDTA were purchased from Hyclone (Los Angeles, CA, United States). Fetal bovine serum was purchased from Biological Industries (Kibbutz Beit Haemek, Israel). Tissue culture plates were obtained from Corning (Corning, NY, USA). RIPA Lysis and Halt Protease and Phosphatase Inhibitor Cocktail $(100 \times)$ were purchased from Thermo Scientific (Rockford, IL, United States) and stored at $4{ }^{\circ} \mathrm{C}$. BCA protein assay kit used to quantify protein concentration was purchased from Beyotime (Shanghai, China). All other chemicals and solvents were of reagent grade or better.

\section{Cell line and cell culture}

C26 cell line was obtained from Shanghai Institute of Materia Medica, Chinese Academy of Sciences. Cells were cultured in RMPI-1640 medium containing $10 \%$ fetal bovine serum at $37^{\circ} \mathrm{C}$ with $5 \% \mathrm{CO}_{2}$.

\section{Animals}

Animals, including both ICR mice used in fatigue experiments and $\mathrm{BALB} / \mathrm{c}$ mice used in cancer cachexia experiments, were purchased from Shanghai SLAC Laboratory Animal Co. Ltd (Shanghai, China). The care and experimental protocols for this study complied with the Chinese regulations and the Guidelines for the Care and Use of Laboratory Animals drawn up by the National Institutes of Health (United States) and were approved by the Institutional Animal Care and Use Committee of the East China Normal University. Mice were maintained on a $12: 12$ light-dark cycle in a temperature-controlled $\left(21-23{ }^{\circ} \mathrm{C}\right)$ and specific pathogen-free conditional room, and were provided with standard rodent chow and water ad libitum. All animals were acclimatized for a week before beginning the study.

\section{Checking of anti-fatigue capacity}

Animal grouping. A total of 18 ICR mice (8 weeks; 26-39 g; half of which were male and half female) were used. Firstly, the exhaustive swimming times (without a load) of the mice were recorded. Then, mice were randomly divided into 3 groups $(n=$ 6 per group; half of which were male and half female) according to their exhaustive swimming time, making the average time equivalent for each group. Mice in the negative control group (CT) were administered with distilled water $(0.1 \mathrm{~mL} / 10 \mathrm{~g}$ body weight, i.g.), and mice in the SBCC treatment group were administered with SBCC at a low dose of $1 \mathrm{~g} \mathrm{~kg}^{-1}$ or at a high dose of $2 \mathrm{~g} \mathrm{~kg}^{-1}$ (once per day for 20 days, i.g.). SBCC was prepared by dissolving in the same volume of distilled water. SBCC could not be fully dissolved in water and thus the mixture was used to treat mice (i.g.). All mice were fed with normal laboratory food and drank water freely. Body weight and food intake were recorded daily.

\section{Exhaustive swimming test}

On the $7^{\text {th }}, 14^{\text {th }}$ and $20^{\text {th }}$ day of treatment, an exhaustive swimming test was conducted similar to previous reports. ${ }^{\mathbf{1 3 , 1 4}}$ Briefly, 30 min after the treatment with SBCC or distilled water, the mice were placed individually in a swimming pool $(50 \mathrm{~cm} \times$ $40 \mathrm{~cm} \times 40 \mathrm{~cm}$ ), filled with water to a depth of $30 \mathrm{~cm}$ and maintained at $22 \pm 1{ }^{\circ} \mathrm{C}$. A lead sinker $(1.5 \%$ of the body weight on $7^{\text {th }}$ day experiment and $3 \%$ of the body weight on $14^{\text {th }}$ and $20^{\text {th }}$ day experiment) was attached to the tail root of each mouse. Exhaustion of the mice was classified as the loss of coordinated movements and inability to rise to the surface within $10 \mathrm{~s}$. The time of exhaustion was recorded and the mice were immediately removed from the pool, dried with a paper towel, and returned to their original cages. The pool water was replaced after each session.

\section{Exhaustive running test}

On the $12^{\text {th }}$ and $18^{\text {th }}$ day of treatment, a treadmill running test was performed similar to previous reports. ${ }^{15,16}$ The exercise protocols were performed with a motor-driven rodent treadmill (YLS-10B, Shandong Academy of Medical Sciences, Yiyan Technology Development Co. Ltd, Shandong, China). The treadmill was equipped with an electric shock grid to provide exercise motivation to the animals by shocking a mouse once it stops running during the experiment. If the mouse is shocked for more than 2 seconds, the runway will stop rotating and once the runway stops rotating 5 times, it is considered as running to exhaustion. Firstly, mice were trained once on the runway adjusted to low difficulty at $15 \mathrm{rpm}$ for $7 \mathrm{~min}$ as adaption. Then, mice were made to run on the treadmill with a set speed of $25 \mathrm{rpm}$ until exhaustion and time to exhaustion was recorded. The instrument parameters settings were: rotating speed $25 \mathrm{rpm}$, current $2 \mathrm{~mA}$, open channel 6, shock endurance time $2 \mathrm{~s}$, experiment hours $2 \mathrm{~h}$, experiment minutes $120 \mathrm{~min}$, time limitation $2 \mathrm{~h}$, breaks 5 times, interval between breaks $50 \mathrm{~s}$. To minimize diurnal effects, all animals were exercised at the same time (09.00-12.00 am).

\section{Forelimb grip test}

On the $17^{\text {th }}$ day of treatment, a forelimb grip test was conducted using a mouse grip tester (YLS-13A, Shandong Academy of 
Medical Sciences, Yiyan Technology Development Co. Ltd, Shandong, China). The grip served as an indicator of the skeletal muscle strength of mice. Measurement was repeated 8 times for each mouse.

\section{Cancer cachexia mice model}

Cancer cachexia in mice was induced as reported in our previous report. ${ }^{17}$ Briefly, a total of 24 male BALB/c mice (6-8 weeks old) were randomly divided into three groups ( 8 mice per group): healthy group without tumor (Health group), C26 tumor-bearing mice group (C26 model group) and C26 tumorbearing mice treated with $\operatorname{SBCC}\left(1.5 \mathrm{~g} \mathrm{~kg}^{-1}\right)$ group (C26 + SBCC group). On day 0 , mice received subcutaneous injections of C26 tumor cells $\left(1.0 \times 10^{6}\right.$ cells $\left./ 0.1 \mathrm{ml}\right)$ in the right flank. Starting from the next day, C26 model group mice were orally administrated with sterile distilled water every day, and SBCCtreated mice received oral administration of SBCC (1.5 g $\mathrm{kg}^{-1}$ ). Body weight, body temperature and food intake were measured daily from C26 inoculation to completion of the study. On day 7, tumors were first detected. The shortest diameter $(x)$ and longest diameter $(y)$ of each tumor were recorded using calipers. Tumor volume was calculated using the following formula: $V=x \times x \times y \times 0.5$. When the mice lost more than $10 \%$ of their body weight or when their tumor volumes reached $2000 \mathrm{~mm}^{3}$, the experiment was ended. All animals were euthanized by $\mathrm{CO}_{2}$ inhalation, and tissues including tumor, gastrocnemius muscle, eWAT (epididymal white fat), inguinal fat tissue, heart and liver were dissected and weighed.

\section{Western blotting}

Western blotting was performed as described previously. ${ }^{\mathbf{1 7}}$ Briefly, the tissues (gastrocnemius muscle or eWAT) were homogenized in RIPA buffer plus a phosphatase protease inhibitor by a tissue lyser $(50 \mathrm{~Hz}, 15 \mathrm{~min})$ bought from QIAGEN. The lysates were centrifuged at $13000 \mathrm{rpm}$ for $30 \mathrm{~min}$ at $4{ }^{\circ} \mathrm{C}$. The supernatant was quantified for protein concentration using a BCA Protein Assay Kit (Beyotime, Shanghai, China). Equal amounts of protein samples were separated by $10 \%$ SDS-PAGE gel electrophoresis and transferred to a polyvinylidene fluoride membrane. The polyvinylidene fluoride membranes were blocked in 5\% non-fat milk in phosphate buffered saline containing $0.1 \%$ Tween 20 (TPBS) for $1 \mathrm{~h}$ at room temperature and then incubated with primary antibodies diluted in 5\% BSATPBS at $4{ }^{\circ} \mathrm{C}$ overnight. The primary antibodies used were as follows: mouse anti-MHC monoclonal antibody (1:1000, DSHB, Iowa City, IA, United States), rabbit anti-MyoD polyclonal antibody (1:1000, Santa Cruz Biotechnology, Dallas, TX, United States), rabbit anti-MyoG polyclonal antibody (1: 500, Santa Cruz Biotechnology, Dallas, TX, United States), rabbit anti-AKT monoclonal antibody $(1: 1000$, Cell Signaling Technology), rabbit anti-p-AKT monoclonal antibody (1 : 1000, Cell Signaling Technology), rabbit anti-AMPK $\alpha$ monoclonal antibody ( 1 : 1000, Cell Signaling Technology), rabbit anti-p-AMPK $\alpha$ monoclonal antibody (1:1000, Cell Signaling Technology), rabbit anti-P38 MAPK monoclonal antibody $(1: 1000$, Cell
Signaling Technology), rabbit anti-p-P38 MAPK monoclonal antibody ( $1: 1000$, Cell Signaling Technology), rabbit antimTOR monoclonal antibody $(1: 1000$, Cell Signaling Technology), rabbit anti-p-mTOR monoclonal antibody (1:1000, Cell Signaling Technology), rabbit anti-ERK polyclonal antibody ( 1 : 1000, Cell Signaling Technology), rabbit anti-p-ERK monoclonal antibody (1:1000, Cell Signaling Technology), rabbit anti-TORC1 monoclonal antibody (1:1000, Cell Signaling Technology), rabbit anti-PGC-1 $\alpha$ monoclonal antibody $(1: 1000$, Cell Signaling Technology), rabbit anti-MuRF-1 monoclonal antibody (1:1000, Abcam), rabbit anti-atrogin-1 monoclonal antibody ( $1: 1000$, Abcam), rabbit anti-HSL polyclonal antibody (1 : 1000, Cell Signaling Technology), rabbit anti-p-HSL polyclonal antibody (1:1000, Cell Signaling Technology), rabbit anti-UCP1 monoclonal antibody (1 : 1000, Cell Signaling Technology) and actin-HRP (1:1000, Santa Cruz Biotechnology). HRP-conjugated goat anti-mouse secondary antibody (1 : 5000, Multi Sciences, Hangzhou, China) and goat anti-rabbit secondary antibody (1:5000, Multi Sciences) were incubated with membranes for $1 \mathrm{~h}$ in $5 \%$ non-fat milk in TPBS. ECL Chemiluminescent Kit (Thermo Fisher, Waltham, MA, United States) was used to visualize the antibody-antigen interaction and chemical luminescence of membranes was detected by an Amersham Imager 600 (GE).

\section{Hematoxylin-eosin (H\&E) staining}

Samples of gastrocnemius muscle and eWAT were freshly isolated and fixed in $4 \%$ paraformaldehyde for $24 \mathrm{~h}$. Paraffinembedded tissues were cut in $10 \mu \mathrm{m}$ sections stained with H\&E by standard procedures. All samples were observed under an Olympus IX70 fluorescence microscope. Ten fields of each image were selected randomly for calculation of adipocyte diameter and myofiber cross-sectional area using Image J software. The shortest diameter $(x)$ and longest diameter $(y)$ of each myofiber were recorded using Image J. The muscle fiber crosssectional area was calculated using the following formula: $V=$ $3.14 \times x \times y \times 0.25$.

\section{Measurement of glycerol, triglyceride and inflammatory cytokines in serum}

The levels of glycerol and triglycerides (TG) were assessed through commercial enzymatic kits (Glycerol Quantification Kit, \#E1002; Triglyceride Quantification Kit, \#E1013, Applygen, Beijing, China, respectively), following the manufacturer's protocols. The levels of TNF- $\alpha$ and IL- 6 in the serum were determined by using ELISA kits (Mouse TNF-alpha High Sensitivity ELISA Kit, \#EK282HS-96; Mouse IL-6 High Sensitivity ELISA Kit, \#EK206HS-96, Multi Sciences, Hangzhou, China) following the manufacturer's protocols.

\section{Statistical analysis}

All values were expressed as mean \pm standard error of the mean (SEM). All statistical analyses were performed using GraphPad Prism 5.0 software. Comparisons among different groups were conducted using one-way ANOVA. A $p$ value less than 0.05 was considered as statistically significant. 


\section{Results}

\section{Effects of SBCC on physical characteristics of normal mice}

As shown in Fig. 1, the food intake of mice was not affected by SBCC treatment (Fig. 1A). Treatment of SBCC (ig. $1 \mathrm{~g} \mathrm{~kg}^{-1}$ or $2 \mathrm{~g}$ $\mathrm{kg}^{-1}$ for 20 days) slightly increased the body weight of mice
(Fig. 1B). The organ indexes of heart (Fig. 1C) and liver (Fig. 1D) were slightly lower. The tissue indexes of muscle (gastrocnemius, Fig. 1E) and fat (eWAT, Fig. 1F) were slightly increased in the SBCC-treated group, compared with the control group. However, the differences between the SBCC-treated group and the control group were all not significant.
A



C

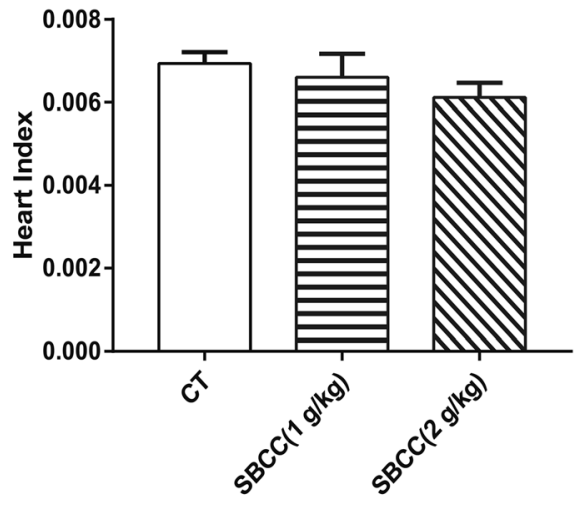

$E$

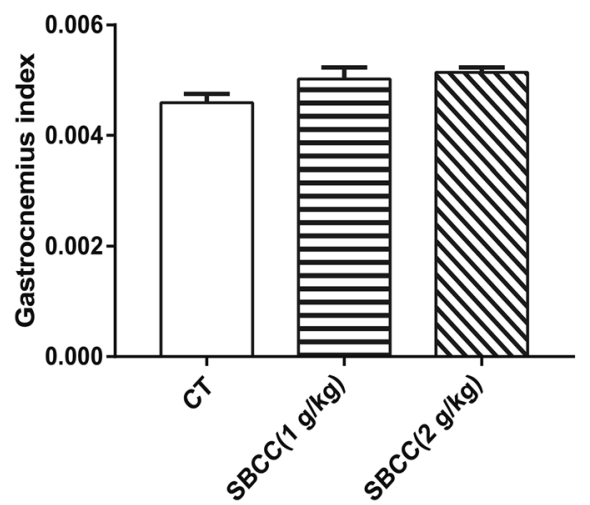

B

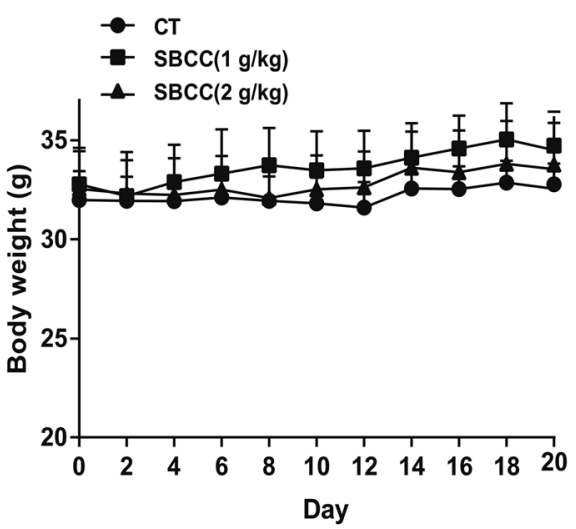

D

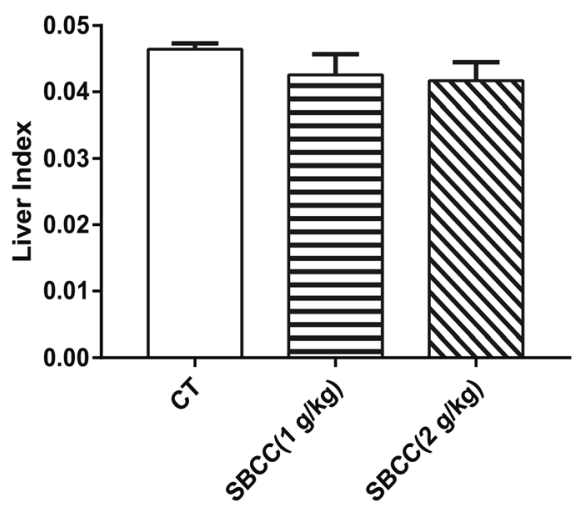

$\mathrm{F}$

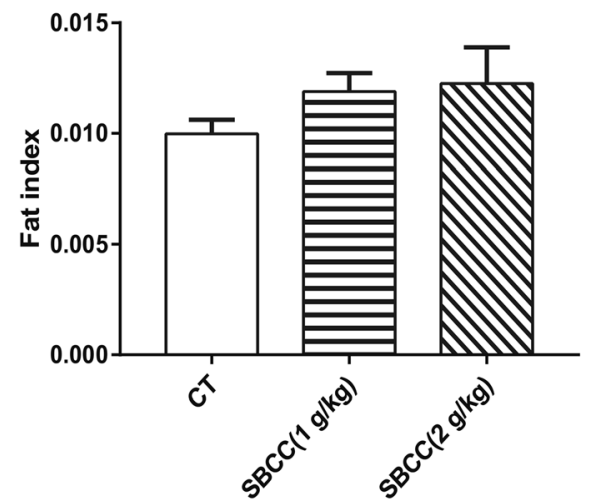

Fig. 1 Effects of SBCC on physical characteristics of mice. ICR mice were treated with distilled water (solvent control) or different dosages of SBCC $\left(1 \mathrm{~g} \mathrm{~kg}^{-1}\right.$ or $\left.2 \mathrm{~g} \mathrm{~kg}^{-1}\right)$ by gavage for 20 days. Body weight and food intake were recorded every other day and the liver, heart, gastrocnemius and fat mass were measured on day 20. (A) Accumulative food intake. (B) Body weight. (C) Heart index. (D) Liver index. (E) Muscle index. (F) Fat index. Values are expressed as mean $\pm \operatorname{SEM}(n=6)$. 


\section{Effects of SBCC on muscle fatigue and muscle strength of mice}

Exhaustive swimming test, exhaustive running test and mouse grip test were carried out to evaluate the anti-fatigue properties of SBCC. As shown in Fig. 2A, SBCC treatment (both $1 \mathrm{~g} \mathrm{~kg}^{-1}$ and $2 \mathrm{~g} \mathrm{~kg}^{-1}$ ) for 20 days could significantly increase the swimming time of mice in the exhaustive swimming test. On day 20, compared with the control group, $1 \mathrm{~g} \mathrm{~kg}^{-1}$ and $2 \mathrm{~g} \mathrm{~kg}^{-1}$ SBCC administration prolonged swimming time by nearly $244.9 \%$ and $319.9 \%$. SBCC treatment at $2 \mathrm{~g} \mathrm{~kg}^{-1}$ could also significantly increase the running time of mice in the exhaustive running test and a significant difference could be observed as early as after only 12 days of SBCC treatment. Treatment of SBCC at $2 \mathrm{~g} \mathrm{~kg}^{-1}$ increased the running time by $93.5 \%$, compared with that of the control mice (Fig. 2B). These results suggested that SBCC could exhibit considerable anti-fatigue activities. Furthermore, as shown in Fig. 2C, SBCC treatment (both $1 \mathrm{~g} \mathrm{~kg}^{-1}$ and $2 \mathrm{~g} \mathrm{~kg}^{-1}$ ) significantly increased the muscle grip strength of mice. On day 17, compared with the control group, $1 \mathrm{~g} \mathrm{~kg}^{-1}$ and $2 \mathrm{~g} \mathrm{~kg}^{-1}$ SBCC improved the forelimb grip by $41.2 \%$ and $37.9 \%$, respectively. The anti-fatigue activities of SBCC might be related to the enhanced strength of muscle of SBCC-treated mice.

\section{Effects of SBCC on histological characteristics and expression} of signal proteins in mouse gastrocnemius muscle tissues

Results of H\&E staining of gastrocnemius muscle in CT and SBCC-treated mice are shown in Fig. 3A (representative results) and Fig. 3B (statistical results). SBCC treatment significantly increased gastrocnemius muscle cross-sectional area. Results of
A

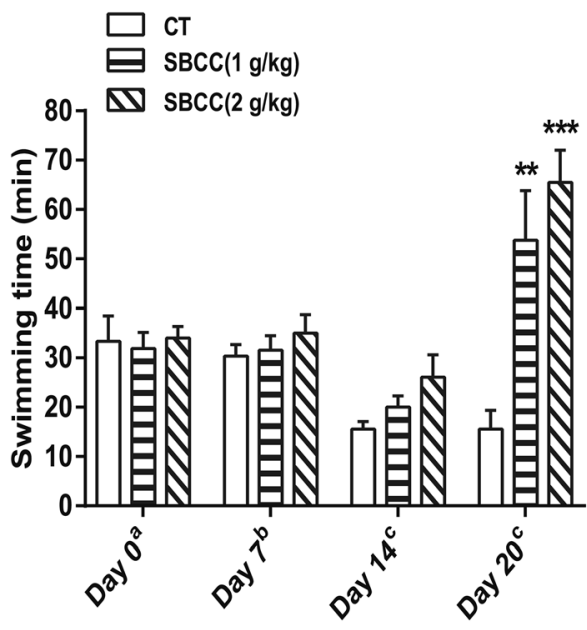

C



B



Fig. 2 Effects of SBCC on induced fatigue and muscle strength of mice. ICR mice were supplemented with distilled water and SBCC (1 and $2 \mathrm{~g} \mathrm{~kg}{ }^{-1}$ ) orally for 20 days. The endurance performance was measured by swimming time to exhaustion on days $0,7,14$ and 20 and running time to exhaustion on days 12 and 18, and the muscle force was measured by forelimb grip test on day 17. (A) Results of exhaustive swimming test: (a) swimming without a load; (b) swimming with load of $1.5 \%$ body weight; (c) swimming with load of $3 \%$ body weight. (B) Results of exhaustive running test. (C) Results of forelimb grip test. Values are expressed as mean $\pm \operatorname{SEM}(n=6)$. ${ }^{*} p<0.05, * * p<0.01, * * * p<0.001$ vs. CT (control) group. 
A

CT

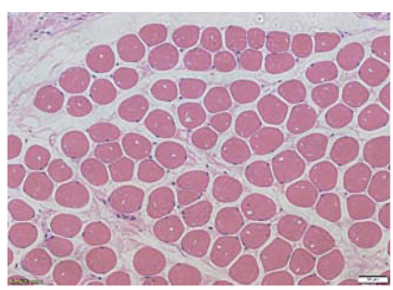

B

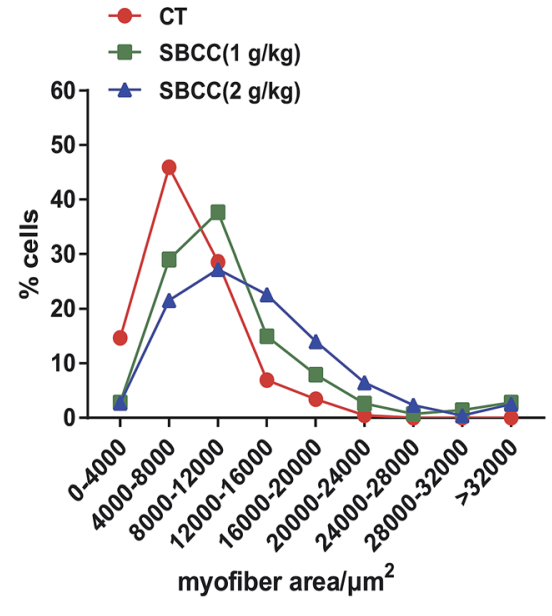

$\operatorname{SBCC}(1 \mathrm{~g} / \mathrm{kg})$

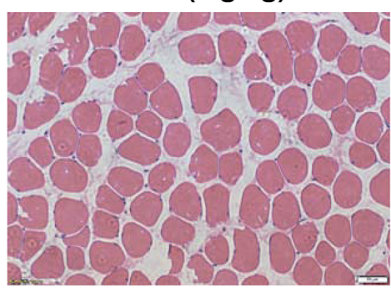

$\operatorname{SBCC}(2 \mathrm{~g} / \mathrm{kg})$

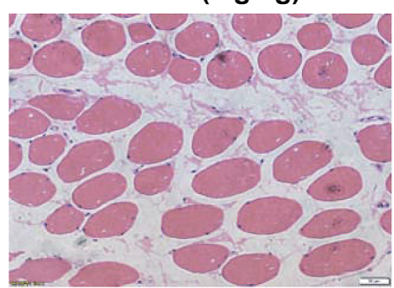

C

$\operatorname{sBCC}(\mathbf{g} / \mathrm{kg})$



D
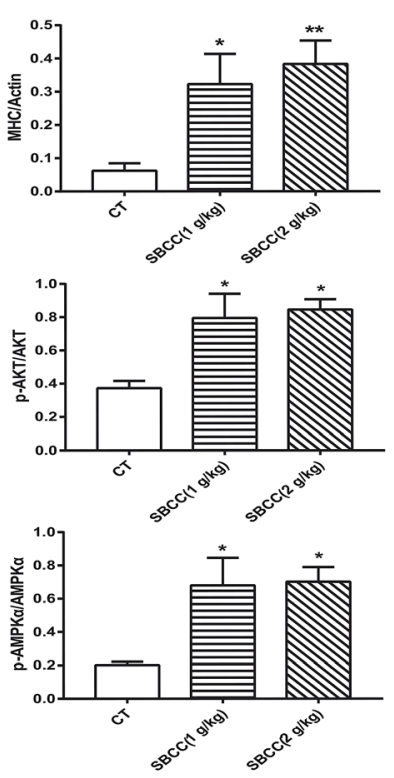
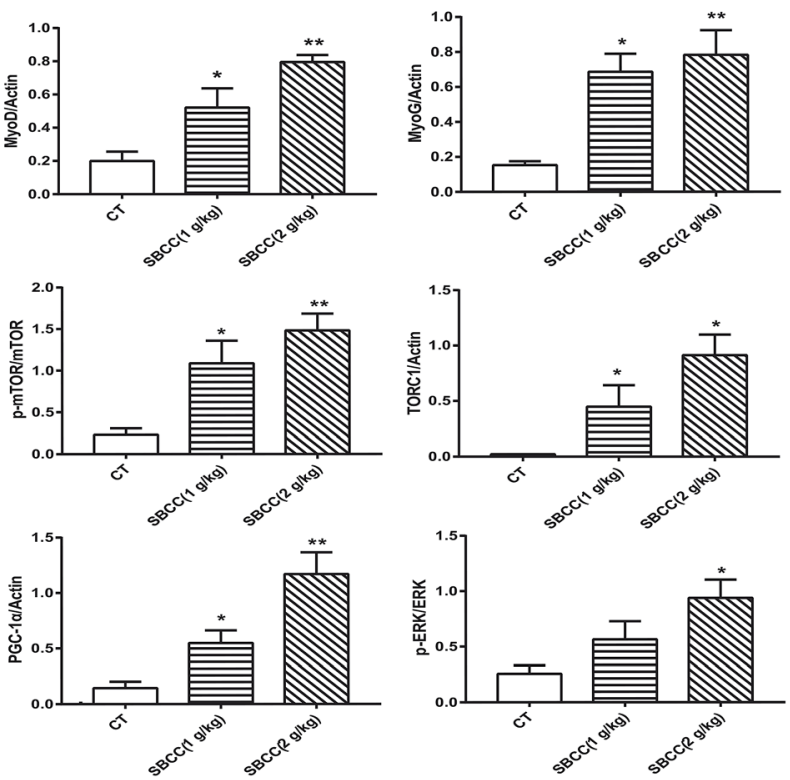

Fig. 3 Effects of SBCC on histological characteristics and expression of signal proteins in mouse gastrocnemius muscle tissues. ICR mice were treated with distilled water and SBCC $\left(1\right.$ and $\left.2 \mathrm{~g} \mathrm{~kg}^{-1}\right)$ by gavage daily for 20 days. The myofiber area and protein expression levels of gastrocnemius muscle were measured with H\&E staining and immunoblot analysis. (A) Representative results of H\&E-stained sections of mouse gastrocnemius muscle. Scale bar $=50 \mu \mathrm{m}$. (B) Statistical results of quantification of the myofiber area of mouse gastrocnemius tissue. (C) Results of western blotting assay of the protein levels of phosphorylated AKT, total AKT, phosphorylated mTOR, total mTOR, phosphorylated AMPK $\alpha$, total AMPK $\alpha$, phosphorylated ERK, total ERK, TORC1, MyoG, MyOD, MHC and PGC-1 $\alpha$ in mouse gastrocnemius. Actin served as loading control. (D) Quantification of (C). Values are expressed as mean \pm SEM. ${ }^{*} p<0.05, * * p<0.01$ vs. CT (control) group. 
A

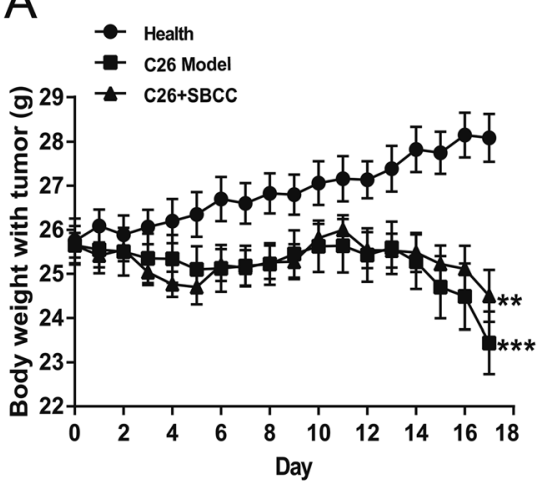

C

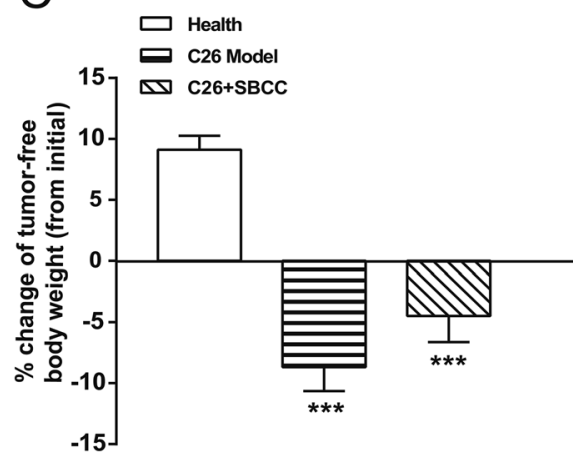

E

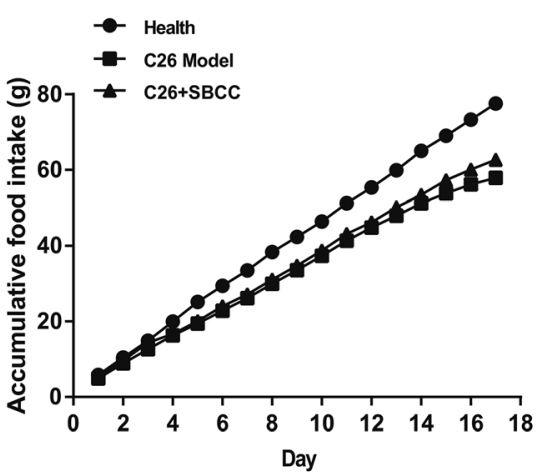

G

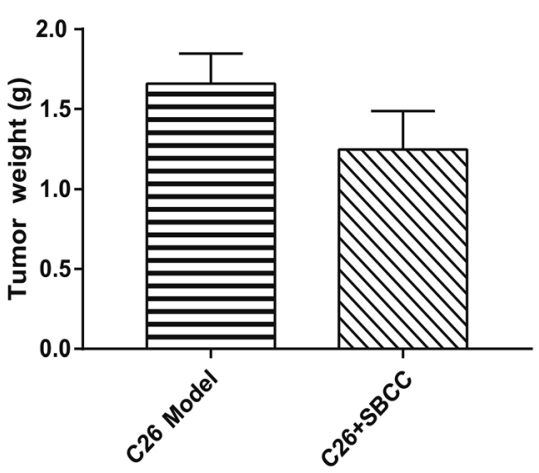

B



D



F



Fig. 4 Effects of SBCC on physiological parameters and tumor growth in mice bearing C26 tumor. Healthy BALB/c mice were supplemented with distilled water (solvent control) and mice bearing C26 tumor were treated with distilled water or SBCC (1.5 g kg kg $^{-1}$ by gavage daily for 17 days. Body weight, body temperature, food intake and tumor volume were recorded every day and tumor weight was measured at the end of the experiment. (A) The body weight curve of mice. (B) The tumor-free body weight curve of mice. (C) Change of tumor-free body weight of mice from the beginning of the experiment to the end of the experiment. (D) The body temperature curve of mice. (E) The accumulative food intake curve of mice. (F) The tumor growth curve of mice. (G) The tumor weight at the end of the experiment. Values are expressed as mean \pm SEM ( $n=$ 8). $* * p<0.01, * * * p<0.001$ versus healthy mice; ${ }^{\# \#} p<0.01$ vs. C26 model mice. 


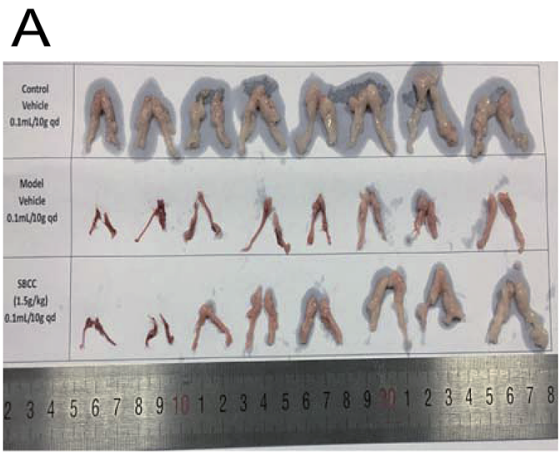

C



E

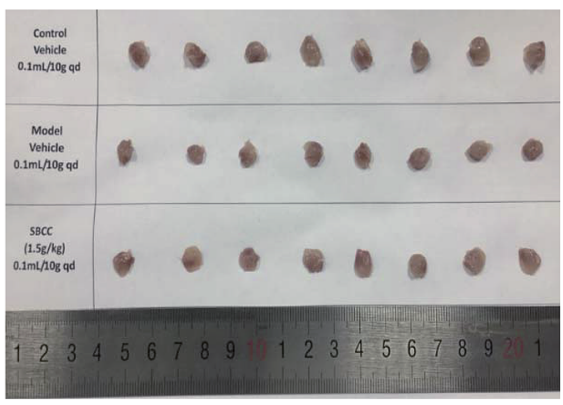

G

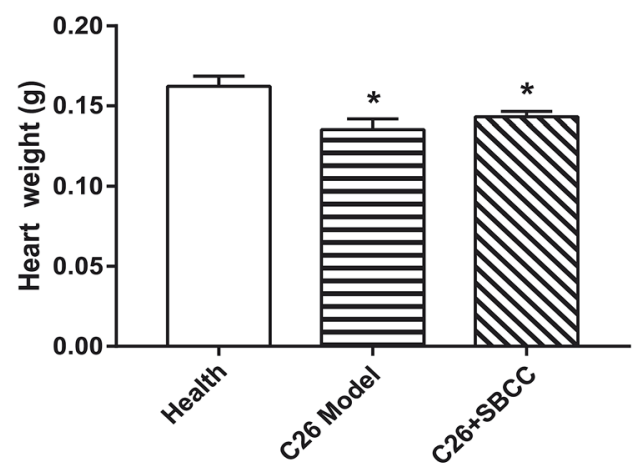

B

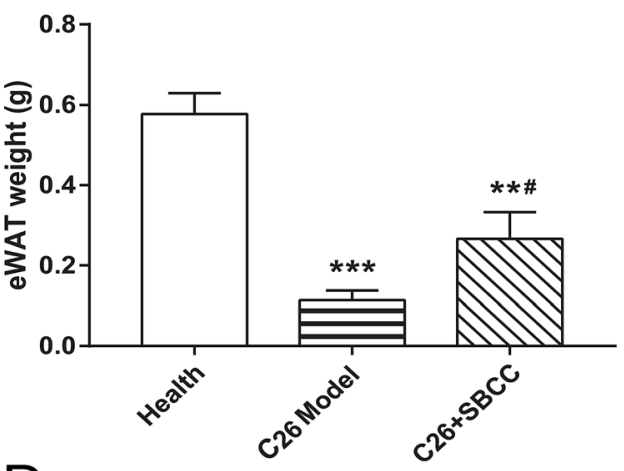

D
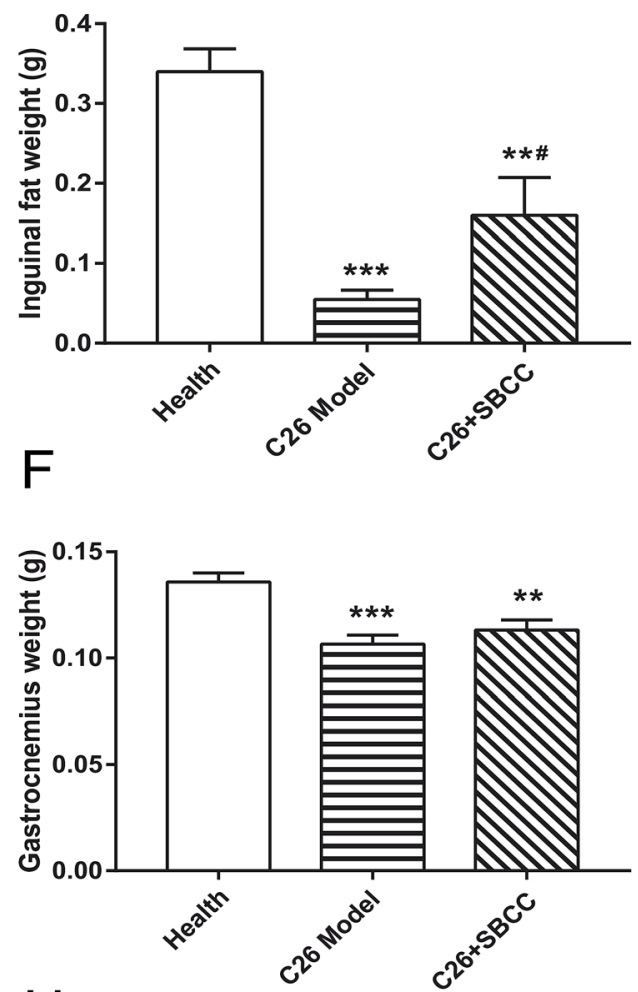

$\mathrm{H}$

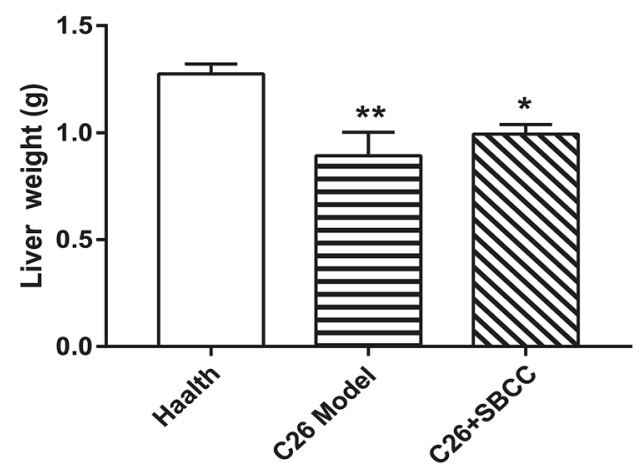

Fig. 5 Effects of SBCC on mass of fat, muscle, heart and liver tissue in mice bearing C26 tumor. Healthy BALB/c mice were supplemented with distilled water (solvent control) and mice bearing C26 tumor were treated with distilled water or SBCC (1.5 g kg $\left.{ }^{-1}\right)$ by gavage daily for 17 days, and the masses of eWAT, inguinal fat tissue, gastrocnemius, heart and liver were measured at the end of the experiment. (A) Photo of isolated eWAT tissues. (B) Weight of eWAT tissues. (C) Photo of isolated inguinal fat tissues. (D) Weight of inguinal fat tissues. (E) Photo of isolated gastrocnemius muscles. (F) Weight of gastrocnemius muscles. (G) Weight of heart tissues. (H) Weight of liver tissues. Values are expressed as mean \pm SEM $(n=8)$. ${ }^{*} p<0.05,{ }^{* *} p<0.01,{ }^{* * *} p<0.001$ vs. health mice; ${ }^{*} p<0.05$ vs. C26 model mice. 
western blotting assay of signal proteins related to muscle myoblast differentiation, protein synthesis and cell metabolism are shown in Fig. 3C (representative results) and Fig. 3D (statistical results). Levels of MHC, MyoD and MyoG were upregulated in SBCC-treated mouse muscle. SBCC treatment induced phosphorylation of AKT, mTOR, AMPK $\alpha$ and ERK, which suggested activation of these pathways. Accordingly, levels of TORC1 and PGC- $1 \alpha$ were also increased in the SBCCtreated group.

\section{Effects of SBCC on the physiological parameters in cancer-} associated cachexia mice

The effects of SBCC on physiological parameters of C26 tumorbearing mice were systematically evaluated and the results are shown in Fig. 4 and 5. As shown in Fig. 4, during the experimental period of 17 days, the body weight (Fig. 4A), tumor-free body weight (Fig. 4B and C), body temperature (Fig. 4D) and food intake (Fig. 4E) decreased markedly in mice bearing C26 tumor (C26 model group), compared with the normal control mice (Health group). As shown in Fig. 4A and B, the body weight of mice in the $\mathrm{C} 26$ model group decreased progressively. As shown in Fig. 4C, the $\mathrm{C} 26$ tumor-bearing mice lost about $10 \%$ of their body weight from the beginning of the experiment to the end of the experiment. As shown in Fig. 5, both the fat mass of eWAT (Fig. 5A and B), inguinal fat weight (Fig. 5C and D) and the mass of gastrocnemius muscle (Fig. 5E and F) decreased markedly in the C26 model mice, compared with the normal control mice. Generally, the international consensus diagnostic criterion for cancer cachexia is weight loss greater than $5 \%$ in the past 6 months and depletion of muscle and fat tissues. ${ }^{\mathbf{1 8 - 2 0}}$ C26 tumor-bearing mice model is a well-accepted animal model of cancer cachexia. ${ }^{19}$ The body weight loss (about $10 \%$ ) of the
A

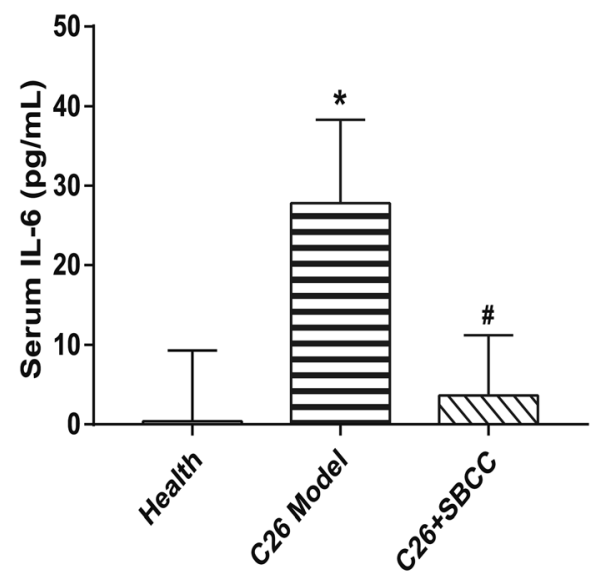

C

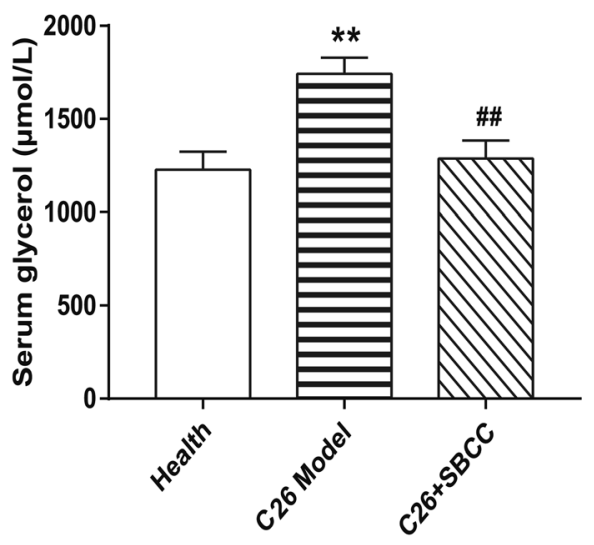

B

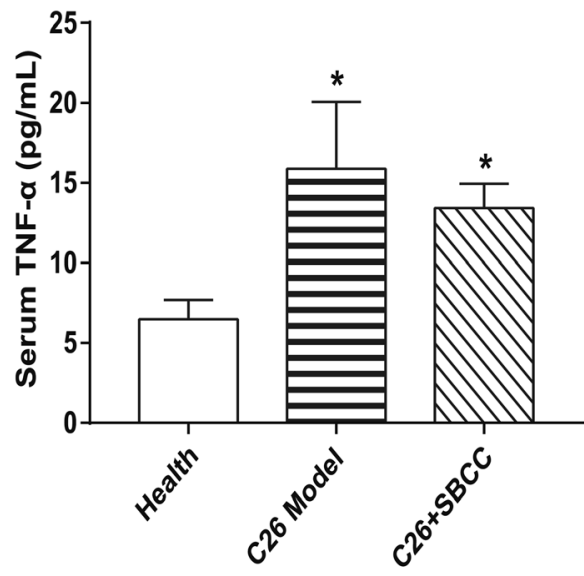

D

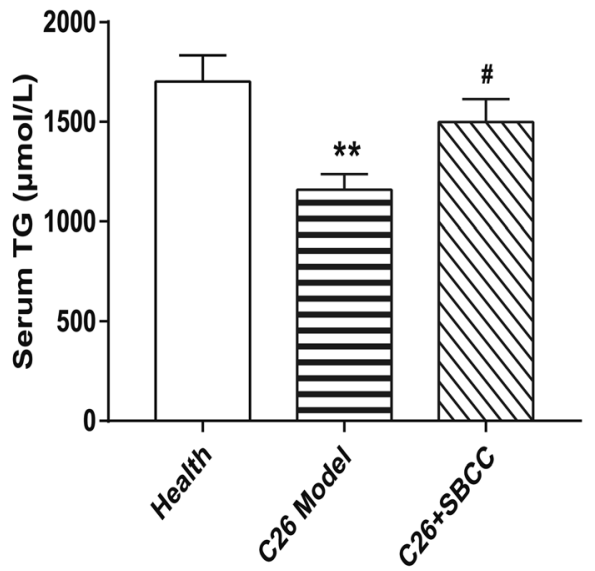

Fig. 6 Effects of SBCC on levels of serum inflammatory cytokines, glycerol and TG in mice bearing C26 tumor. Healthy mice were supplemented with distilled water and mice bearing C26 tumor were treated with distilled water or SBCC $\left(1.5 \mathrm{~g} \mathrm{~kg}^{-1}\right)$ by gavage daily for 17 days. The serum concentrations of TNF- $\alpha$, IL- 6 , glycerol and triglycerides in mice on day 17 were determined by ELISA kits and commercial enzymatic kits according to the manufacturer's protocols. (A) Serum level of IL-6. (B) Serum level of TNF- $\alpha$. (C) Serum level of glycerol. (D) Serum level of TG. Data presented are the mean $\pm \operatorname{SEM}(n=8) .{ }^{*} p<0.05,{ }^{* *} p<0.01 \mathrm{vs}$. health mice; ${ }^{\#} p<0.05,{ }^{\# \#} p<0.01$ vs. C26 model mice. 
A

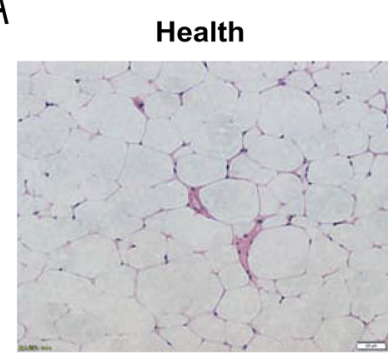

B

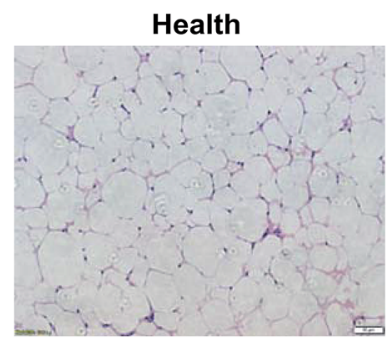

C26 Model

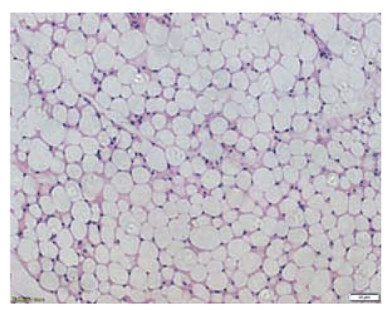

C26+SBCC
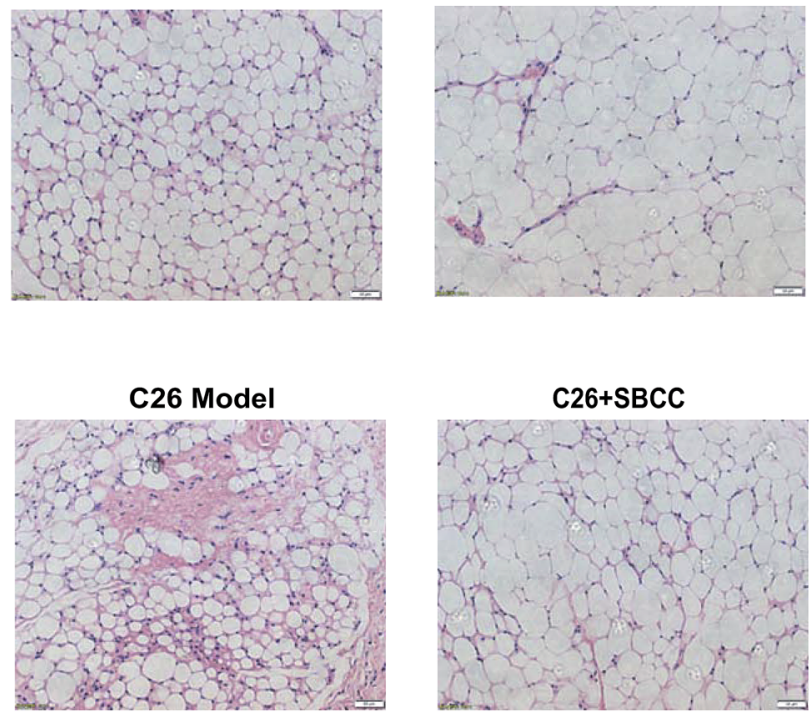

$\mathrm{C} 26+\mathrm{SBCC}$



C



$\mathrm{F}$
D

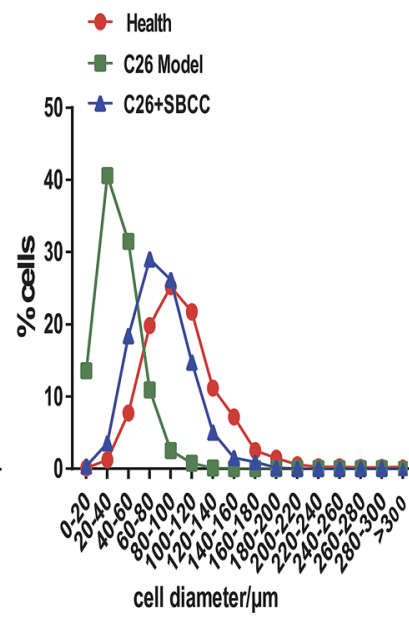

E

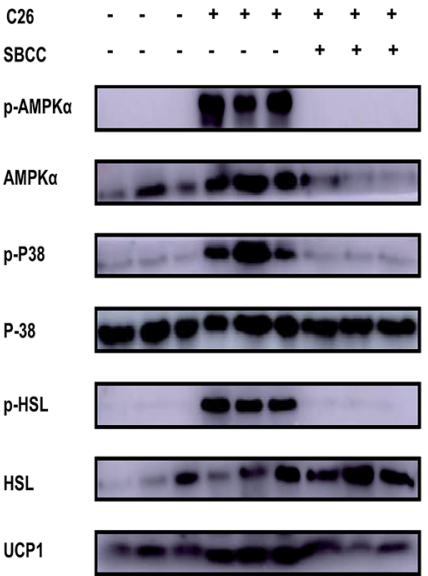

Actin

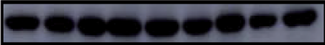

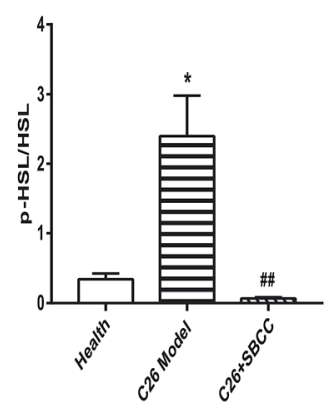


Fig. 7 Effects of SBCC on histochemical characteristics and levels of signal proteins in fat tissues of C26 cancer cachexia mice. Healthy mice were supplemented with distilled water and mice bearing C26 tumor were treated with distilled water or SBCC $\left(1.5 \mathrm{~g} \mathrm{~kg}^{-1}\right)$ by gavage daily for 17 days. The diameters of adipocytes and protein expression levels in eWAT tissue were measured with H\&E staining and immunoblot analysis. (A) Representative H\&Estained sections of mouse eWAT tissues. Scale bar $=50 \mu \mathrm{m}$. (B) Representative H\&E-stained sections of mouse inguinal fat tissue. Scale bar $=50 \mu \mathrm{m}$. (C) Quantitative results of the adipocyte diameter in mouse eWAT tissues. (D) Quantitative results of the adipocyte diameter in mouse inguinal fat tissues. (E) Representative western blotting assay results of phosphorylated AMPK $\alpha$, total AMPK $\alpha$, phosphorylated p38 MAPK, total p38 MAPK, phosphorylated HSL, total HSL and UCP1 in the eWAT tissue of mice with or without treatment of SBCC. Actin served as loading control. (F) Quantification of (E). Data presented are the mean \pm SEM of three independent experiments. ${ }^{*} p<0.05,{ }^{* *} p<0.01,{ }^{* * *} p<0.001$ vs. health mice; ${ }^{\#} p<0.05,{ }^{\# \#} p<0.01,{ }^{\# \# \#} p<0.001$ vs. C26 model mice. 
A



B

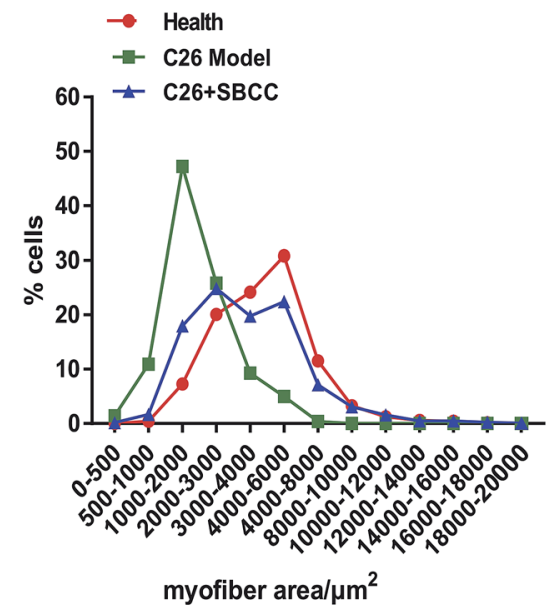

C26 Model

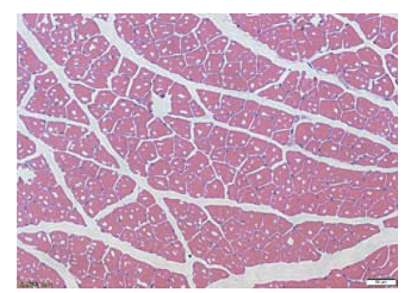

C
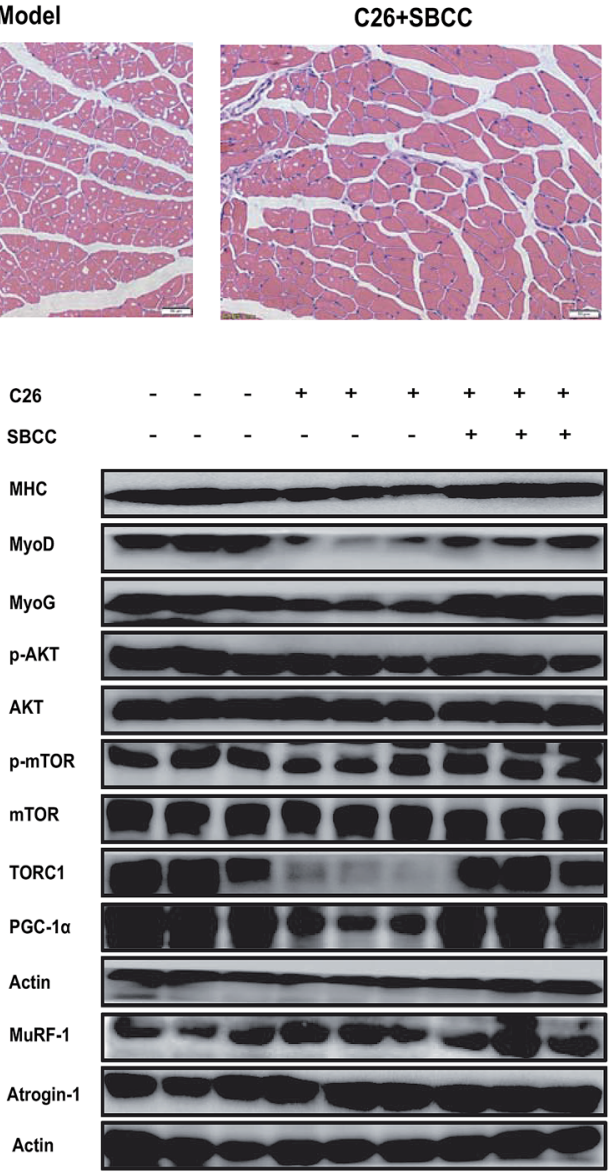

D
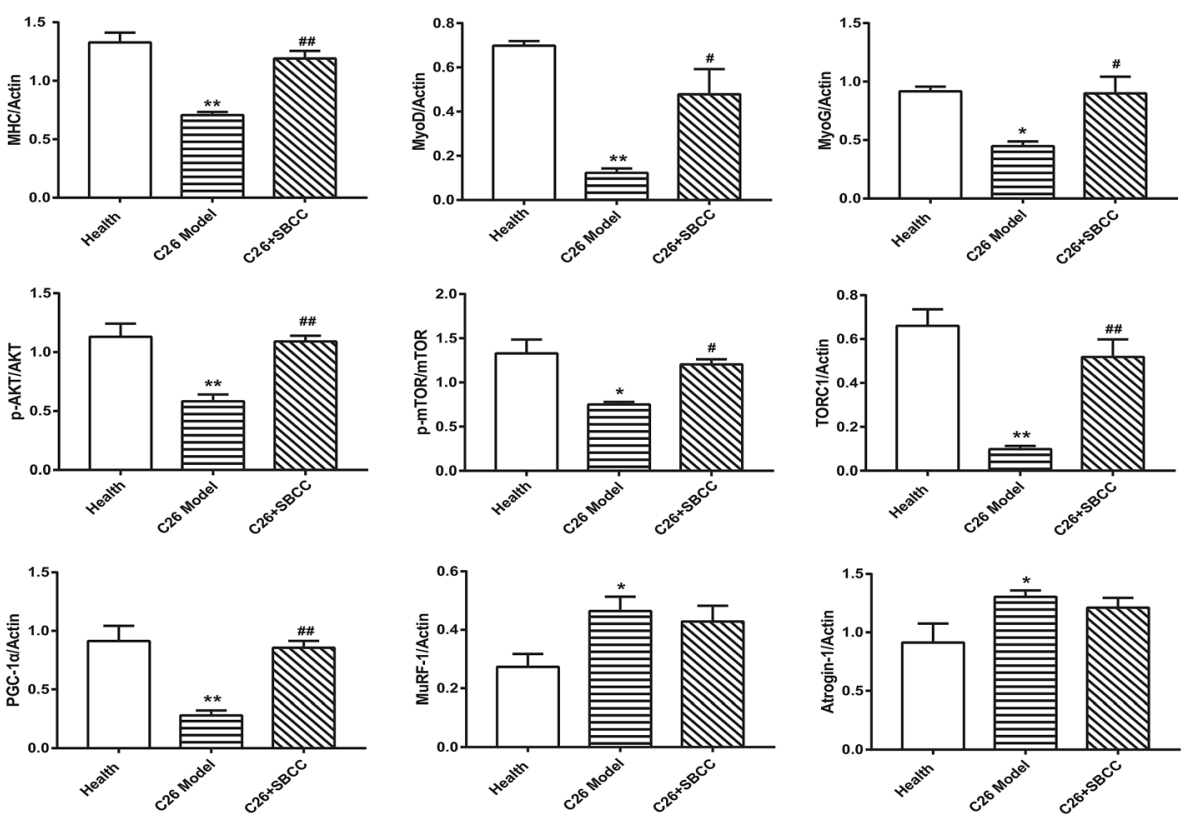

Fig. 8 Effects of SBCC on histological characteristics and expression of signal proteins in gastrocnemius muscle tissues of C26 cancer cachexia mice. Healthy mice were supplemented with distilled water and mice bearing C26 tumor were treated with distilled water or SBCC $\left(1.5 \mathrm{~g} \mathrm{~kg}{ }^{-1}\right)$ by gavage daily for 17 days. The myofiber area and protein expression level in gastrocnemius muscle were measured with H\&E staining and immunoblot analysis. (A) Representative H\&E-stained sections of mice gastrocnemius muscle cells. Scale bar $=50 \mu \mathrm{m}$. (B) Quantitative results of the myocyte area of mice gastrocnemius muscle tissues. (C) Representative western blotting assay results of phosphorylated AKT, total AKT, phosphorylated mTOR, total mTOR, TORC1, MyoG, MyoD, MHC, PGC-1 $\alpha$, MuRF-1 and atrogin-1 in the gastrocnemius muscle tissues. Actin served as loading control. (D) Quantification of (C). Data presented are the mean \pm SEM of three independent experiments. ${ }^{*} p<0.05,{ }^{* *} p<0.01$ vs. health mice; ${ }^{*} p<0.05,{ }^{\# \#} p<0.01$ vs. C26 model mice. 


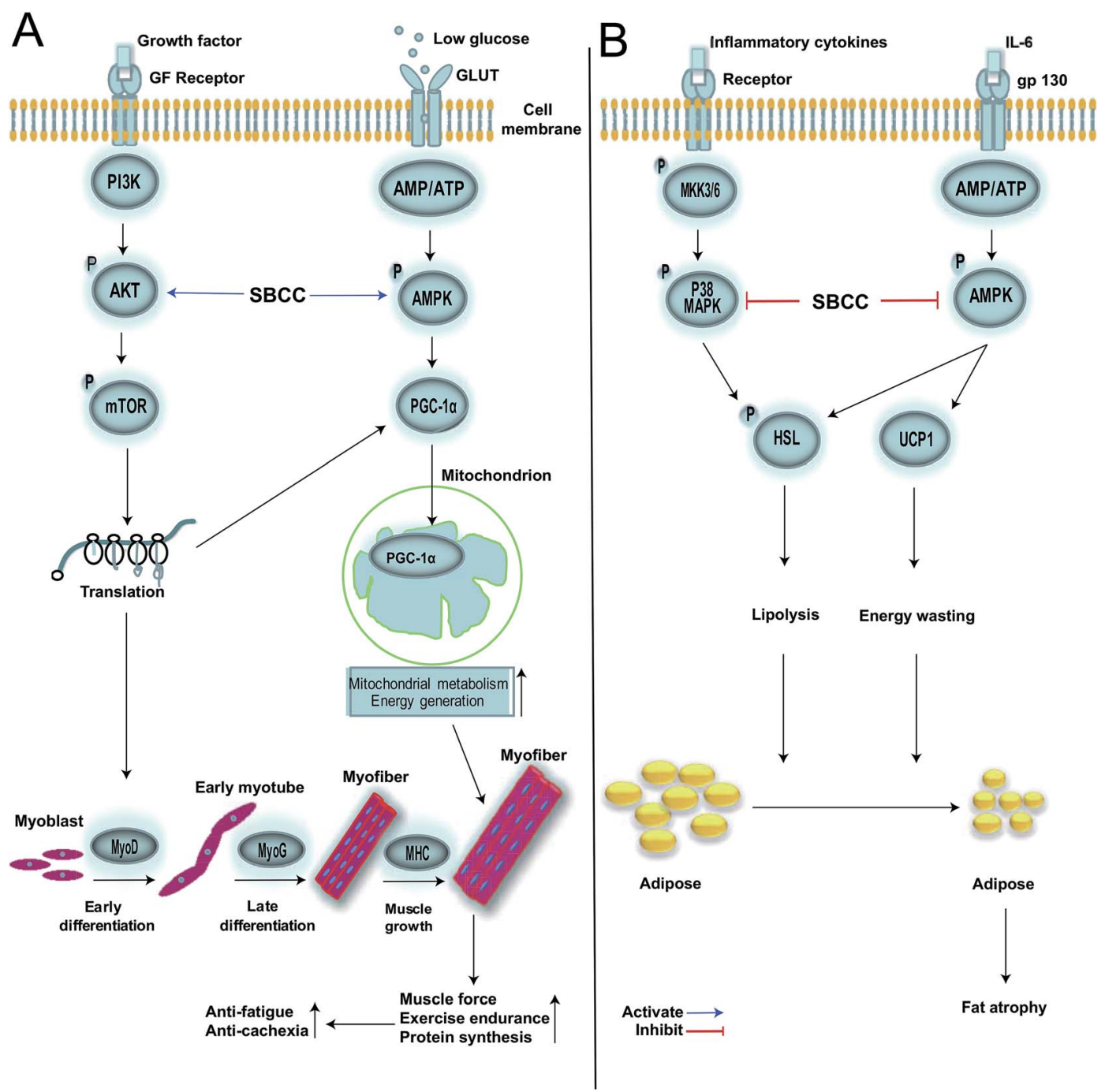

Fig. 9 Illustration of signal networks by which SBCC exhibited its effects in muscle and fat. (A) Signal network in myocytes. SBCC exhibits antifatigue and anti-cachexia activities by activating the AKT/mTOR pathway and AMPK/PGC-1 $\alpha$ pathway in myocytes. Firstly, SBCC leads to activation of the AKT/mTOR protein synthesis pathway and up-regulation in the expression of MyoD, MyoG, MHC and PGC-1 $\alpha$. MyoD promotes myoblast differentiation into early myotube, and then MyoG causes myotube fusion into myofiber. The increase of MHC accelerates myofiber growth, resulting in the enhancement of muscle force. Secondly, SBCC activates the AMPK/PGC-1 $\alpha$ pathway in myocytes. Long-time exercise leads to a shortage of glucose and increase of the ratio of AMP/ATP in muscle cells, which activates the AMPK/PGC-1 $\alpha$ pathway. PGC-1 $\alpha$ is transported into mitochondria to boost mitochondrial metabolism and energy generation, resulting in the improvement of exercise endurance. (B) Signal network in adipocytes. SBCC exhibits anti-cachexia activity by inhibiting the p38 MAPK pathway and AMPK pathway in adipocytes. Many intracellular signals in fat tissue in cancer-cachexia mice are activated by inflammatory cytokines which lead to the phosphorylation of p38 MAPK. IL- 6 bonding to gp130 can affect fat cell glucose intake by insulin resistance, which activates p-AMPK to thermogenesis and energy wasting by increasing the expression of UCP1. The activation of the p38 MAPK pathway and AMPK pathway phosphorylates HSL to lipolysis. SBCC inhibits these pathways to increase the fat mass in cancer-cachexia mice.

C26 tumor-bearing mice and the decrease in weight of muscle tissue and fat tissue were in accordance with previous reports using $\mathrm{C} 26$ tumor cachexia model ${ }^{19}$ and confirmed the success in establishing a cancer cachexia animal model in the present study.

SBCC treatment only slightly increased the body weight and tumor-free body weight of mice but significantly increased the body temperature of mice. SBCC slightly increased the food intake amount of mice bearing C26 tumor (Fig. 4E). Interestingly, tumor growth, as shown by both tumor volume (Fig. 4F) and tumor weight (Fig. 4G), was somewhat inhibited in SBCC- treated mice though the differences were not significant. Notably, SBCC treatment could significantly increase the eWAT mass and inguinal fat mass in C26 tumor-bearing mice though it could not ameliorate the decrease in muscle mass. SBCC treatment showed no influence on decrease in heart mass and liver mass in C26 tumor-bearing mice (Fig. 5G and H).

\section{Effects of SBCC on levels of serum inflammatory cytokines, glycerol and TG in cancer-associated cachexia mice}

As shown in Fig. 6, significant elevation of IL-6 and TNF- $\alpha$ levels was observed in serum of C26 tumor-bearing mice. Notably, 
SBCC treatment could significantly decrease the IL-6 serum level (Fig. 6A) but not the TNF- $\alpha$ level (Fig. 6B). Furthermore, the levels of glycerol and TG in mice serum were also determined. Increase of glycerol (Fig. 6C) and decrease of TG (Fig. 6D) were observed in C26 tumor-bearing mice, which suggested enhanced lipolysis in mice with cachexia. Importantly, SBCC treatment could significantly ameliorate the decrease of TG and increase of glycerol.

\section{Effects of SBCC on histochemical characteristics and levels of signal proteins in fat tissues}

As shown in Fig. 7A and C, the size of adipocyte cell diameter decreased in C26 cachexia model mice, and SBCC treatment could ameliorate the decrease of adipocyte cell diameter in eWAT tissue. Similar results could be found for inguinal fat tissue (Fig. 7B and D). Furthermore, results of western blotting assay of signal proteins in eWAT tissue are shown in Fig. 7E (representative results) and Fig. $7 \mathrm{~F}$ (statistical results). As shown in Fig. 7E and F, phosphorylation activation of AMPK $\alpha$ and p38 MAPK was observed in C26 tumor-bearing mice, but SBCC treatment could inhibit the activation of AMPK and p38 MAPK pathways. Phosphorylation of hormone-sensitive lipase (HSL), a rate-limiting enzyme whose phosphorylation on Ser559/660 is crucial in regulating adipocyte lipolysis, was also observed in C26 tumor-bearing mice. However, the phosphorylation of HSL could be inhibited by SBCC treatment. Furthermore, SBCC also inhibited the expression of UCP1, which blocked adipose tissue ineffective thermogenesis. These results suggested that SBCC administration might ameliorate eWAT loss in cancer cachexia through the modulation of AMPK and p38 MAPK pathways and keys actors in the lipolytic and lipid utilization pathways.

\section{Effects of SBCC on histochemical characteristics and levels of signal proteins in gastrocnemius muscle tissues}

Although SBCC treatment could not significantly inhibit the decrease in muscle mass in cachexia mice, it could partly ameliorate the decrease of gastrocnemius myofiber area in C26 cachexia model mice (Fig. 8A and B). SBCC could also modify the changes of signal protein levels related to muscle growth and metabolism in gastrocnemius muscle of $\mathrm{C} 26$ tumor-bearing mice. As shown in Fig. 8C and D, expression levels of MHC, MyoD, MyoG, TORC1, and PGC- $1 \alpha$ decreased in C26 model mice. The AKT/mTOR pathway, which is related to protein synthesis in muscle tissue, was inhibited in C26 model mice. SBCC treatment ameliorated the decrease in MHC, MyoD, MyoG, TORC1, and PGC- $1 \alpha$ and enhanced AKT and mTOR pathway. At the same time, the protein degradation pathway, as shown by the significantly up-regulated expression levels of MuRF-1 and atrogin-1, was activated in C26 model mice. SBCC treatment could not inhibit the increase in MuRF-1 and atrogin1 expression. These results suggested that SBCC administration might facilitate myoblast protein synthesis in muscle tissues of cancer cachexia mice through activation of AKT-mTOR pathway but it exhibited no influence on protein degradation of muscle tissues.

\section{Discussion}

In modern society, the rapid rhythm of life leads to reduced exercise, and elevated work stress makes people prone to fatigue. In turn, fatigue seriously affects work efficiency and quality of life. Furthermore, diseases such as cancer could also cause fatigue. ${ }^{21,22}$ Notably, use of mushrooms and related products as functional foods for treatment of fatigue has been well accepted. Edible medicinal mushrooms probably mitigate human fatigue through effects on various functional systems including the muscular, cardiovascular, hormone, and immune systems. ${ }^{23}$ Among the medical mushrooms, "Cordyceps fungi" are some of the most important species. The name "Cordyceps" comes from the Latin words cord and ceps, meaning "club" and "head", respectively. The most famous family member in the Cordyceps genus is Cordyceps sinensis (DongChongXiaCao), which is well known as one of the three greatest invigorants together with Panax and Pilose antlers. ${ }^{1}$ Different mycelial strains have been isolated from natural Cordyceps, manufactured in large quantity by fermentation technology and used as medicament products or health-care products. ${ }^{1}$ SBCC, a functional food product containing fermentation of Cordyceps fungi, is popularly used in China to reduce fatigue though its effects and mechanisms have not been systemically studied.

Results of the present study suggested that SBCC could exhibit considerable anti-fatigue activities in mice. It significantly improved muscle resistance, thus increasing the exhaustive swimming and running times of mice in forced swimming test and exhaustive running test, respectively. These results were in accordance with previous reports about antifatigue activities of Cordyceps fungi. ${ }^{1,24-26}$ Our results also showed that SBCC increased the strength of skeletal muscle. The effects of SBCC might be related to promotion of myoblast differentiation, protein synthesis and cell metabolism through activation of the AKT, mTOR and AMPK signaling pathways. The increase of MHC protein level and gastrocnemius muscle cross-sectional area in SBCC-treated mice implied enhanced muscle growth in the tissue. And, levels of MyoD and MyoG were significantly up-regulated in gastrocnemius tissue of mice treated with SBCC in a dose-dependent manner. The results suggested that SBCC induced both skeletal muscle myoblast differentiation and growth. In muscle cells, the AKT pathway is involved in cell viability, proliferation and differentiation. ${ }^{27}$ By regulating the mTOR signal pathway, AKT controls protein synthesis, cell differentiation and thus muscle hypertrophy or atrophy. ${ }^{27,28}$ Therefore, by activating the AKT-mTOR pathway, SBCC might induce the expression of myogenic regulatory factors MyoD and MyoG and thus increase the levels of musclespecific genes such as MHC, the major structural protein in myotubes. AMPK plays a major role in regulating cellular energy and control of metabolism. ${ }^{29}$ AMPK interacts with mTOR in regulation of cellular and organismal energy. ${ }^{30}$ Our results showed that SBCC induced activation of AMPK and mTOR and increased the level of PGC- $1 \alpha$ which was favorable for muscle endurance. PGC- $1 \alpha$ is a master regulator of muscular phenotypic changes and aerobic performance and it is critical in 
determining the response of muscle to stimuli such as exercise training. ${ }^{31}$ PGC- $1 \alpha$ could be regulated by $\mathrm{AMPK}^{32}$ and mTOR pathways. $^{33}$ SBCC supplementation could activate the AMPK and mTOR pathways and induce elevation of PGC- $1 \alpha$ level. Thus, SBCC exhibited considerable anti-fatigue effects and the effects might be related to increased muscle endurance based on enhancement of muscle cell growth and differentiation and improvement of muscle response to exercise training by activating AKT, mTOR, and AMPK pathways.

In the present study, the effects of SBCC on cancer-related cachexia were also observed to check the possibility of using SBCC as a functional food for patients with cancers. Cancer cachexia is a kind of whole-body metabolic disorder syndrome accompanied by severe wasting of muscle and adipose tissue. Notably, up to now, there is no approved therapeutic agent for the treatment or prevention of cancer cachexia. ${ }^{17}$ Results of the present study showed that SBCC treatment could partly ameliorate cachexia symptoms of mice bearing C26 tumor. SBCC significantly ameliorates the decrease in body temperature in cachexia mice and slightly ameliorates the decrease in body weight. In cachexia mice, the AKT-mTOR pathway is inhibited, resulting in the decrease of protein synthesis and muscle atrophy. ${ }^{34}$ Muscle-specific E3 ubiquitin ligases such as MuRF-1 and atrogin-1 are involved in muscle atrophy of cancer cachexia. ${ }^{35}$ In the present study, a decrease in protein synthesis, as shown by inhibition of the AKT/mTOR pathway, and an increase in protein degradation, as shown by up-regulated expression of MuRF-1 and atrogin-1, were observed in C26 tumor-bearing mice. SBCC treatment could significantly ameliorate the decrease in protein synthesis but could not inhibit the increase in protein degradation of cachexia mice. The decrease in myofiber area was also ameliorated in SBCCtreated cachexia mice though SBCC could not significantly inhibit the loss of muscle weight in cachexia mice. On the contrary, fat tissues reacted better to the effects of SBCC. SBCC significantly inhibited the decrease in fat tissue. The values of both eWAT tissue weight and inguinal fat tissue weight were significantly higher in SBCC-treated mice, compared with C26 model mice. And, results of histological analysis showed that SBCC increased the size of adipocyte cell diameter of tumorbearing mice. In addition, SBCC inhibited the decrease of TG level and increase of glycerol level in serum of cachexia mice, which suggested the protective effects of SBCC on fat tissue against lipolysis.

Inflammatory cytokines such as IL-6 secreted by tumors have been well established to play an important role in the pathogenesis of cancer cachexia, especially fat loss through lipolysis. IL-6 could act as a tumorkine in provoking catabolic changes in adipose tissue which result in lipolysis and atrophy. ${ }^{36}$ Importantly, our results showed that SBCC significantly inhibited the increase of IL-6 in serum of cachexia mice. Therefore, the protective effects of SBCC on fat tissue against lipolysis might be related to decreased serum IL-6 level in SBCC-treated mice. As shown in results of western blotting assay of signal proteins in fat tissues of C26 model mice, activation of AMPK and p38MAPK was observed in cachexia fat tissues. And, phosphorylation of HSL, which is a rate-limiting enzyme in regulating adipocyte lipolysis, was induced in C26 model mice. HSL is a substrate for AMPK; thus activation of AMPK could increase phosphorylation of HSL to mobilize lipids and induce lipolysis ${ }^{37}$ and AMPK inhibitors such as compound $\mathrm{C}$ could partially abrogate lipolysis of 3T3-L1 adipocytes. ${ }^{38}$ What is more, p38 MAPK signaling was also reported to phosphorylate HSL in pancreatic cancer exosome-induced adipose tissue lipolysis. ${ }^{39}$ SBCC treatment inhibited the activation of AMPK and p38MAPK in cachexia fat tissue, decreased the level of phosphorylated HSL and thus ameliorated lipolysis in fat tissue. Furthermore, IL-6 in cachexia could induce the increase of UCP1 level which uncoupled mitochondrial respiration toward thermogenesis instead of ATP synthesis, leading to increased lipid mobilization and energy expenditure in cachectic mice. ${ }^{40}$ Our results also showed high UCP1 level in fat tissues of C26 model mice. SBCC treatment might decrease lipid mobilization and energy expenditure by inhibiting the increase of UCP1 expression. The influence of SBCC on UCP1 level might be related to decreased serum IL-6 level in SBCC-treated mice.

Different components in SBCC such as Cordyceps polysaccharide, cordycepic acid, cordycepin and amino acids might work synergistically to exhibit anti-fatigue and anti-cachexia activities. Previous reports indicated that polysaccharides extracted from Cordyceps fungi distinctly enhanced the exercise endurance of mice. ${ }^{41}$ Cordyceps polysaccharides could protect cultured cells from hydrogen peroxide-induced mitochondrial dysfunction by decreasing reactive oxygen species (ROS) production $^{42}$ and also could protect cells from tumor neurosis factor- $\alpha$ induced mitochondrial abnormality and apoptosis. ${ }^{43}$ Cordycepic acid and cordycepin could ameliorate the LPSinduced inflammatory response of cultured cells. ${ }^{44}$ Cordycepin treatment also could reduce the generation of ROS. ${ }^{45}$ Amino acids might contribute to protein synthesis in tissues and protect tissues from atrophy. For example, arginine activates the AMPK/ PGC-1 $\alpha$ pathway and mTOR pathway to promote skeletal muscle fiber type transformation and enhance protein synthesis. ${ }^{46} \mathrm{~L}^{-}$ Arginine enhances protein synthesis by phosphorylating mTOR in $\mathrm{C} 2 \mathrm{C} 12$ cells. ${ }^{47}$ Methionine regulates mTORC1 via the ERK1/2 signal transduction process in $\mathrm{C} 2 \mathrm{C} 12$ cells. ${ }^{48}$ Arginine, L-citrulline and branched-chain amino acids could protect muscle cells from wasting in vitro in an mTORC1-dependent manner. ${ }^{49-51}$ Based on reported activities of the components, we could only speculate that Cordyceps polysaccharides, cordycepic acid, cordycepin and amino acids in SBCC might all contribute to its antifatigue and anti-cachexia activities. Further work identifying the active components of SBCC involved in its anti-fatigue and anticachexia activities is necessary.

In summary, results of the present study provided evidence that oral administration of SBCC could increase muscle endurance and exhibit anti-fatigue activities. Previous clinical studies showed that Cordyceps sinensis could augment the performance of long-distance runners and anti-fatigue ability of healthy young volunteers as compared to placebo control. ${ }^{52,53}$ Moreover, Cordyceps militaris improved the resistance of elderly healthy volunteers to fatigue over the placebo group in doubleblind, placebo-controlled studies. ${ }^{\mathbf{5 4 , 5 5}}$ Our results were in accordance with clinical anti-fatigue efficacy of Cordyceps fungi 
products. Furthermore, SBCC was capable of ameliorating cancer-associated cachexia by attenuating fat tissue atrophy, possibly by reducing the inflammatory cytokine IL-6 secretion, regulating over-lipolysis and decreasing lipid over-utilization. SBCC treatment might be conducive to the improvement of the quality of life of patients with advanced cancer through decreasing release of inflammatory cytokines, alleviating lipolysis of fat tissue, promoting skeletal muscle growth and inhibiting decrease of body temperature. To the best of our knowledge, this is the first report showing the ameliorating effects of Cordyceps fungi products on cancer-associated cachexia. No clinical use of anti-cachexia effects of Cordyceps fungi products has been reported before. Although further studies are required to check more Cordyceps fungi products and confirm their anti-cachexia activities, the findings in the present study suggested that Cordyceps fungi might be worth developing as adjuvant treatment agents for therapy of cancer cachexia. The possible signal networks by which SBCC exerted its anti-fatigue and anti-cachexia effects in myocytes and adipocytes are shown in Fig. 9A and B, respectively. Our results supported the anti-fatigue use of SBCC for people with poor health and also suggested its potential use in patients with cancer cachexia.

\section{Conflicts of interest}

There are no conflicts to declare.

\section{Abbreviations}

SBCC SiBaoChongCao

MHC Myosin heavy chain

MyoD Myogenic differentiation antigen

MyoG Myogenic regulatory factors

PGC- Peroxisome proliferator-activated receptor gamma

$1 \alpha \quad$ coactivator $1 \alpha$

HSL Hormone-sensitive lipase

UCP1 Uncoupling protein 1

eWAT Epididymal white fat

TG Triglyceride

\section{Acknowledgements}

This work was partly supported by the National Natural Science Foundation of China (No. 81873056, 81872496, 21572263 and 21572067), the pilot project of the Chinese Academy of Sciences (XDA12020356), the Shanghai Science \& Technology Innovation Action Program (no. 15140904800), and the Chen-Kai-Xian Academician Workstation in Yunnan Province (no. 2017IC041).

\section{Notes and references}

1 X. Zhou, Z. Gong, Y. Su, J. Lin and K. Tang, Cordyceps fungi: natural products, pharmacological functions and developmental products, J. Pharm. Pharmacol., 2009, 61, 279-291.

2 T. T. Zuo, Y. L. Li, H. Y. Jin, F. Gao, Q. Wang, Y. D. Wang and S. C. Ma, HPLC-ICP-MS speciation analysis and risk assessment of arsenic in Cordyceps sinensis, Chin. Med., 2018, 13, 19.

3 X. Wei, H. Hu, B. Zheng, Z. Arslan, H. C. Huang, W. Mao and Y. M. Liu, Profiling metals in Cordyceps sinensis by using inductively coupled plasma mass spectrometry, Anal. Methods, 2017, 9, 724-728.

4 P. Yang, X. X. Zhao and Y. W. Zhang, Comparison and review on specifications of fermented Cordyceps sinensis products, China J. Chin. Mater. Med., 2018, 43, 463-468.

5 L. H. Chen, Y. Wu, Y. M. Guan, C. Jin, W. F. Zhu and M. Yang, Analysis of the High-Performance Liquid Chromatography Fingerprints and Quantitative Analysis of Multicomponents by Single Marker of Products of Fermented Cordyceps sinensis, J. Anal. Methods Chem., 2018, 2018, 5943914.

6 Y. Li, C. Lin, W. B. Xu, X. Y. Zhang, Y. Z. Wang, H. Gao, X. J. Dou, X. Y. Hao and Y. G. Zhang, Effects of Acremonium terricola culture on growth performance, antiocidant and immune indexes of serum and liver in Sprague Dawley rats, Chin. J. Anim. Nutr., 2016, 28, 9.

7 A. Chi, H. Li, C. Kang, H. Guo, Y. Wang, F. Guo and L. Tang, Anti-fatigue activity of a novel polysaccharide conjugates from Ziyang green tea, Int. J. Biol. Macromol., 2015, 80, 566-572.

8 J. Liu, C. Du, Y. Wang and Z. Yu, Anti-fatigue activities of polysaccharides extracted from Hericium erinaceus, Exp. Ther. Med., 2015, 9, 483-487.

9 D. Toomey, H. P. Redmond and D. Bouchier-Hayes, Mechanisms mediating cancer cachexia, Cancer, 1995, 76, 2418-2426.

10 A. M. Shum and P. Polly, Cancer cachexia: molecular targets and pathways for diagnosis and drug intervention, Endocr., Metab. Immune Disord.: Drug Targets, 2012, 12, 247-259.

11 C. Ming-Hua, Z. Bao-Hua and Y. Lei, Mechanisms of Anorexia Cancer Cachexia Syndrome and Potential Benefits of Traditional Medicine and Natural Herbs, Curr. Pharm. Biotechnol., 2016, 17, 1147-1152.

12 K. C. Cheng, Y. X. Li and J. T. Cheng, The use of herbal medicine in cancer-related anorexia/cachexia treatment around the world, Curr. Pharm. Des., 2012, 18, 4819-4826.

13 H. A. Oh, D. E. Kim, H. J. Choi, N. J. Kim and D. H. Kim, Antifatigue Effects of 20(S)-Protopanaxadiol and 20(S)Protopanaxatriol in Mice, Biol. Pharm. Bull., 2015, 38, 1415-1419.

14 B. Qi, L. Liu, H. Zhang, G. X. Zhou, S. Wang, X. Z. Duan, X. Y. Bai, S. M. Wang and D. Q. Zhao, Anti-fatigue effects of proteins isolated from Panax quinquefolium, $J$. Ethnopharmacol., 2014, 153, 430-434.

15 M. Belviranli, H. Gokbel, N. Okudan and K. Basarali, Effects of grape seed extract supplementation on exercise-induced oxidative stress in rats, Br. J. Nutr., 2012, 108, 249-256.

16 W. M. Southern, A. S. Nichenko, D. D. Shill, C. C. Spencer, N. T. Jenkins, K. K. McCully and J. A. Call, Skeletal muscle metabolic adaptations to endurance exercise training are 
attainable in mice with simvastatin treatment, PLoS One, 2017, 12, e0172551.

17 C. Miao, Y. Lv, W. Zhang, X. Chai, L. Feng, Y. Fang, X. Liu and X. Zhang, Pyrrolidine Dithiocarbamate (PDTC) Attenuates Cancer Cachexia by Affecting Muscle Atrophy and Fat Lipolysis, Front. Pharmacol., 2017, 8, 915.

18 P. W. Emery, Cachexia in experimental models, Nutrition, 1999, 15, 600-603.

19 A. Bonetto, J. E. Rupert, R. Barreto and T. A. Zimmers, The Colon-26 Carcinoma Tumor-bearing Mouse as a Model for the Study of Cancer Cachexia, J. Visualized Exp., 2016, 117, e54893.

20 M. Sadeghi, M. Keshavarz-Fathi, V. Baracos, J. Arends, M. Mahmoudi and N. Rezaei, Cancer cachexia: Diagnosis, assessment, and treatment, Crit. Rev. Oncol. Hematol., 2018, 127, 91-104.

21 H. Mohandas, S. K. Jaganathan, M. P. Mani, M. Ayyar and G. V. Rohini Thevi, Cancer-related fatigue treatment: An overview, J. Cancer Res. Ther., 2017, 13, 916-929.

22 S. A. Christensen Holz and S. R. Smith, Cancer-Related Fatigue: What You Need to Know, Arch. Phys. Med. Rehabil., 2017, 98, 1717-1718.

23 P. Geng, K. C. Siu, Z. Wang and J. Y. Wu, Antifatigue Functions and Mechanisms of Edible and Medicinal Mushrooms, BioMed Res. Int., 2017, 2017, 9648496.

24 Y. F. Xu, Effect of Polysaccharide from Cordyceps militaris (Ascomycetes) on Physical Fatigue Induced by Forced Swimming, Int. J. Med. Mushrooms, 2016, 18, 1083-1092.

25 J. Song, Y. Wang, M. Teng, G. Cai, H. Xu, H. Guo, Y. Liu, D. Wang and L. Teng, Studies on the Antifatigue Activities of Cordyceps militaris Fruit Body Extract in Mouse Model, Evid.-Based Complementary Altern. Med., 2015, 2015, 174616.

26 W. Yan, T. Li, J. Lao, B. Song and Y. Shen, Anti-fatigue property of Cordyceps guangdongensis and the underlying mechanisms, Pharm. Biol., 2013, 51, 614-620.

27 D. C. Guttridge, Signaling pathways weigh in on decisions to make or break skeletal muscle, Curr. Opin. Clin. Nutr. Metab. Care, 2004, 7, 443-450.

28 E. Erbay and J. Chen, The mammalian target of rapamycin regulates $\mathrm{C} 2 \mathrm{C} 12$ myogenesis via a kinase-independent mechanism, J. Biol. Chem., 2001, 276, 36079-36082.

29 D. Carling, AMPK signalling in health and disease, Curr. Opin. Cell Biol., 2017, 45, 31-37.

$30 \mathrm{~K}$. Inoki, J. Kim and K. L. Guan, AMPK and mTOR in cellular energy homeostasis and drug targets, Annu. Rev. Pharmacol. Toxicol., 2012, 52, 381-400.

31 Y. Kim, M. Triolo and D. A. Hood, Impact of Aging and Exercise on Mitochondrial Quality Control in Skeletal Muscle, Oxid. Med. Cell. Longevity, 2017, 2017, 3165396.

32 D. V. Popov, E. A. Lysenko, I. V. Kuzmin, V. Vinogradova and A. I. Grigoriev, Regulation of PGC-1alpha Isoform Expression in Skeletal Muscles, Acta Naturae, 2015, 7, 48-59.

33 X. Wang, N. Huang, M. Yang, D. Wei, H. Tai, X. Han, H. Gong, J. Zhou, J. Qin, X. Wei, H. Chen, T. Fang and H. Xiao, FTO is required for myogenesis by positively regulating mTOR-PGC-1alpha pathway-mediated mitochondria biogenesis, Cell Death Dis., 2017, 8, e2702.
34 K. C. Fearon, D. J. Glass and D. C. Guttridge, Cancer cachexia: mediators, signaling, and metabolic pathways, Cell Metab., 2012, 16, 153-166.

35 L. Yuan, J. Han, Q. Meng, Q. Xi, Q. Zhuang, Y. Jiang, Y. Han, B. Zhang, J. Fang and G. Wu, Muscle-specific E3 ubiquitin ligases are involved in muscle atrophy of cancer cachexia: an in vitro and in vivo study, Oncol. Rep., 2015, 33, 22612268.

36 M. Tsoli, M. M. Swarbrick and G. R. Robertson, Lipolytic and thermogenic depletion of adipose tissue in cancer cachexia, Semin. Cell Dev. Biol., 2016, 54, 68-81.

37 S. J. Kim, T. Tang, M. Abbott, J. A. Viscarra, Y. Wang and H. S. Sul, AMPK Phosphorylates Desnutrin/ATGL and Hormone-Sensitive Lipase To Regulate Lipolysis and Fatty Acid Oxidation within Adipose Tissue, Mol. Cell. Biol., 2016, 36, 1961-1976.

38 J. T. Hwang, S. H. Kim, M. S. Lee, S. H. Kim, H. J. Yang, M. J. Kim, H. S. Kim, J. Ha, M. S. Kim and D. Y. Kwon, Anti-obesity effects of ginsenoside Rh2 are associated with the activation of AMPK signaling pathway in 3T3-L1 adipocyte, Biochem. Biophys. Res. Commun., 2007, 364, 1002-1008.

39 G. Sagar, R. P. Sah, N. Javeed, S. K. Dutta, T. C. Smyrk, J. S. Lau, N. Giorgadze, T. Tchkonia, J. L. Kirkland, S. T. Chari and D. Mukhopadhyay, Pathogenesis of pancreatic cancer exosome-induced lipolysis in adipose tissue, Gut, 2016, 65, 1165-1174.

40 M. Petruzzelli, M. Schweiger, R. Schreiber, R. CamposOlivas, M. Tsoli, J. Allen, M. Swarbrick, S. Rose-John, M. Rincon, G. Robertson, R. Zechner and E. F. Wagner, A switch from white to brown fat increases energy expenditure in cancer-associated cachexia, Cell Metab., 2014, 20, 433-447.

41 F. Yan, B. Wang and Y. Zhang, Polysaccharides from Cordyceps sinensis mycelium ameliorate exhaustive swimming exercise-induced oxidative stress, Pharm. Biol., 2014, 52, 157-161.

42 W. Shen, D. Song, J. Wu and W. Zhang, Protective effect of a polysaccharide isolated from a cultivated Cordyceps mycelia on hydrogen peroxide-induced oxidative damage in PC12 cells, Phytother. Res., 2011, 25, 675-680.

43 H. Tang, W. Wei, W. Wang, Z. Zha, T. Li, Z. Zhang, C. Luo, H. Yin, F. Huang and Y. Wang, Effects of cultured Cordyceps mycelia polysaccharide A on tumor neurosis factor-alpha induced hepatocyte injury with mitochondrial abnormality, Carbohydr. Polym., 2017, 163, 43-53.

44 Y. Y. Ouyang, Z. Zhang, Y. R. Cao, Y. Q. Zhang, Y. Y. Tao, C. H. Liu, L. M. Xu and J. S. Guo, Effects of cordyceps acid and cordycepin on the inflammatory and fibrogenic response of hepatic stellate cells, Chin. J. Hepatol., 2013, 21, 275-278.

45 C. Dou, Z. Cao, N. Ding, T. Hou, F. Luo, F. Kang, X. Yang, H. Jiang, Z. Xie, M. Hu, J. Xu and S. Dong, Cordycepin Prevents Bone Loss through Inhibiting Osteoclastogenesis by Scavenging ROS Generation, Nutrients, 2016, 8, 231.

46 X. Chen, Y. Guo, G. Jia, G. Liu, H. Zhao and Z. Huang, Arginine promotes skeletal muscle fiber type 
transformation from fast-twitch to slow-twitch via Sirt1/ AMPK pathway, J. Nutr. Biochem., 2018, 61, 155-162.

47 R. Wang, H. Jiao, J. Zhao, X. Wang and H. Lin, L-Arginine Enhances Protein Synthesis by Phosphorylating mTOR (Thr 2446) in a Nitric Oxide-Dependent Manner in C2C12 Cells, Oxid. Med. Cell. Longevity, 2018, 2018, 7569127.

48 Y. Zhou, J. Ren, T. Song, J. Peng and H. Wei, Methionine Regulates mTORC1 via the T1R1/T1R3-PLCbeta-Ca(2+)ERK1/2 Signal Transduction Process in C2C12 Cells, Int. J. Mol. Sci., 2016, 17, 1684.

49 D. J. Ham, M. K. Caldow, G. S. Lynch and R. Koopman, Arginine protects muscle cells from wasting in vitro in an mTORC1-dependent and NO-independent manner, Amino Acids, 2014, 46, 2643-2652.

50 D. J. Ham, B. G. Gleeson, A. Chee, D. M. Baum, M. K. Caldow, G. S. Lynch and R. Koopman, L-Citrulline Protects Skeletal Muscle Cells from Cachectic Stimuli through an iNOSDependent Mechanism, PLoS One, 2015, 10, e0141572.

51 E. H. Herningtyas, Y. Okimura, A. E. Handayaningsih, D. Yamamoto, T. Maki, K. Iida, Y. Takahashi, H. Kaji and K. Chihara, Branched-chain amino acids and arginine suppress MaFbx/atrogin-1 mRNA expression via mTOR pathway in C2C12 cell line, Biochim. Biophys. Acta, 2008, 1780, 1115-1120.

52 T. Hiyoshi, F. Akasu, M. Yoshisugu and M. Fujiwara, Supplemental effects of Cordyceps sinensis extract on long distance runners, Jpn. J. Phys. Fitness Sports Med., 1996, 45, 205-210.

53 A. Nagata, T. Tajima and S. Moriyasu, Effectiveness on ingestion with Cordyceps sinensis drinking during running exercise of humans, Japan Society of Exercise and Sports Physiology, 2002, 9, 85-92.

54 X. Yi, H. Xi-zhen and Z. Jia-shi, Randomized double-blind placebo-controlled clinical trial and assessment of fermentation product of Cordyceps sinensis (Cs-4) in enhancing aerobic capacity and respiratory function of the healthy elderly volunteers, Chin. J. Integr. Med., 2004, 10, 187-192.

55 S. Chen, Z. Li, R. Krochmal, M. Abrazado, W. Kim and C. B. Cooper, Effect ofCs-4 (Cordyceps sinensis) on exercise performance in healthy older subjects: a double-blind, placebo-controlled trial, J. Altern. Complement. Med., 2010, 16, 585-590. 